\title{
Coexistence of Singlet and Triplet Attractive Channels in the Pairing Interactions Mediated by Antiferromagnetic Fluctuations
}

\author{
Hiroshi Shimahara \\ Department of Quantum Matter Science, ADSM, Hiroshima University, Higashi-Hiroshima 739-8526, Japan
}

(Received March 2000)

\begin{abstract}
We propose a phase diagram of quasi-low-dimensional type II superconductors in parallel magnetic fields, when antiferromagnetic fluctuations contribute to the pairing interactions. We point out that pairing interactions mediated by antiferromagnetic fluctuations necessarily include both singlet channels and triplet channels as attractive interactions. Usually, a singlet pairing is favored at zero field, but a triplet pairing occurs at high fields where the singlet pairing is suppressed by the Pauli paramagnetic pair-breaking effect. As a result, the critical field increases divergently at low temperatures. A possible relation to experimental phase diagrams of a quasi-one-dimensional organic superconductor is briefly discussed. We also discuss a possibility that a triplet superconductivity is observed even at zero field.
\end{abstract}

PACS numbers: 74.70.Kn, 74.20.Mn, 74.20.-z

Pairing interactions mediated by a spin fluctuations have been discussed as a possible mechanism of anisotropic superconductivity [1] 9] in exotic superconductors such as organic superconductors, heavy fermion superconductors, high- $T_{\mathrm{c}}$ cuprate superconductors, and ruthenate superconductors. For example, in a ruthenate superconductor $\mathrm{Sr}_{2} \mathrm{RuO}_{4}$, pairing interaction mediated by ferromagnetic fluctuations is a candidate for the mechanism of the superconductivity. The superconductivity in this compound is considered to be due to triplet pairing from experimental results such as those obtained in Knight shift measurements [10] and $\mu S R$ experiments 11.

On the other hand, in the organics, cuprates, and heavy fermion superconductors, anisotropic singlet pairing intereactions mediated by antiferromagnetic fluctuations have been examined by many authors, because these compounds are in proximities to antiferromagnetic phases. However, in some of the quasi-one-dimensional organic superconductors and the heavy fermion superconductors, triplet superconductivities have been supported by some experimental results 12,13]. In particular, in some of the heavy fermion superconductors, the triplet pairing seems to be established.

In a quasi-one-dimensional organic superconductor (TMTSF $)_{2} \mathrm{PF}_{6}$, triplet pairing is supported by recent Knight shift measurements by Lee et al. [13], but it may appear to contradict the proximity to an antiferromagnetic phase. We will briefly discuss later that it does not necessarily contradict the mechanism of superconductivity with pairing interactions mediated by antiferromagnetic fluctuations. There is another contradiction between the thermal conductivity measurements [12] and the NMR experiments [14,15] in (TMTSF) ${ }_{2} \mathrm{ClO}_{4}$. The former indicates a nodeless gap, while the latter suggests an existence of line nodes. However, this contradiction can be consistently explained also in terms of the pair- ing interactions mediated by antiferromagnetic fluctuations $\sqrt{16}$.

In the cuprate high- $T_{\mathrm{c}}$ superconductors, experiments with $\pi$-junction suggest an anisotropic pairing which is conventionally called a $d$-wave pairing. Pairing interactions mediated by spin fluctuations have been discussed by many authors since the discovery of the high- $T_{\mathrm{c}}$ superconductors, for example, Miyake et al. [5] and the present author et al. [6]. However, it is not established to what extent the spin fluctuations contribute to the pairing interactions.

In this paper, we discuss a phase diagram of quasi-lowdimensional type II superconductors on a $T$ - $H$ plane. We consider a situation in which a singlet pairing is favored at zero field because of pairing interactions induced by the exchange of antiferromagnetic spin fluctuations. We restrict our discussion to parallel magnetic fields so that we can regard the orbital pair-breaking effect as weak.

The pairing interactions mediated by spin fluctuations are written as

$$
H^{\prime}=-g \sum_{i j} \chi_{i j} \vec{S}_{i} \cdot \vec{S}_{j}
$$

where $i$ and $j$ denote lattice sites and $\chi_{i j}$ is the expression of the spin susceptibility in real space. The dynamical effects and strong coupling effects can be taken into account by developing a perturbation theory in more basic models such as Hubbard models [6 8]. However, since it is not essential for the purpose of this paper, we discuss pairing interactions of the form of eq. (1) for a while. Equation (1) can be rewritten as

$$
\begin{aligned}
H^{\prime}= & -\frac{g}{N} \sum_{\mathbf{k k}^{\prime}} \chi\left(\mathbf{k}-\mathbf{k}^{\prime}\right)\left[\frac { 1 } { 4 } \left(\psi_{11}^{\dagger}(\mathbf{k}) \psi_{11}\left(\mathbf{k}^{\prime}\right)\right.\right. \\
& \left.+\psi_{1,-1}^{\dagger}(\mathbf{k}) \psi_{1,-1}\left(\mathbf{k}^{\prime}\right)+\psi_{10}^{\dagger}(\mathbf{k}) \psi_{10}\left(\mathbf{k}^{\prime}\right)\right) \\
& \left.-\frac{3}{4} \psi_{00}^{\dagger}(\mathbf{k}) \psi_{00}\left(\mathbf{k}^{\prime}\right)\right]
\end{aligned}
$$


where

$$
\begin{aligned}
\psi_{11}(\mathbf{k}) & =c_{-\mathbf{k} \uparrow} c_{\mathbf{k} \uparrow} \\
\psi_{1,-1}(\mathbf{k}) & =c_{-\mathbf{k} \downarrow} c_{\mathbf{k} \downarrow} \\
\psi_{10}(\mathbf{k}) & =\frac{1}{\sqrt{2}}\left(c_{-\mathbf{k} \uparrow} c_{\mathbf{k} \downarrow}+c_{-\mathbf{k} \downarrow} c_{\mathbf{k} \uparrow}\right) \\
\psi_{00}(\mathbf{k}) & =\frac{1}{\sqrt{2}}\left(c_{-\mathbf{k} \uparrow} c_{\mathbf{k} \downarrow}-c_{-\mathbf{k} \downarrow} c_{\mathbf{k} \uparrow}\right) .
\end{aligned}
$$

When the antiferromagnetic fluctuations are strong, the factor $\chi_{i j}$ changes its sign depending on the distance between the sites $i$ and $j$. In general, the factor $\chi_{i j}$ is positive for the sites $(i, j)$ on the same sublattice, while $\chi_{i j}$ is negative for the sites $(i, j)$ on the different sublattices, for the antiferromagnetic correlations. Here, it is easily verified from eq. (2) that the spin correlations between the different sublattices $\left(\chi_{i j}<0\right)$ favor singlet pairing, while they on the same sublattices $\left(\chi_{i j}>0\right)$ favor triplet pairing.

For example, in tight binding models near the halffilling, the factor $\chi_{i j}$ changes its sign alternately. For the nearest-neighbor sites $(i, j), \chi_{i j} \equiv \bar{\chi}_{1}<0$, while for the next-nearest-neighbor sites $\chi_{i j} \equiv \bar{\chi}_{2}>0$. The former $\bar{\chi}_{1}<0$ favor the singlet pairing, while the latter $\bar{\chi}_{2}>0$ contribute to the next-nearest-neighbor triplet pairing.

The triplet pairing interactions between the nextnearest-neighbor sites have not been considered to be important so far, because the nearest-neighbor singlet pairing interactions are usually much stronger than the next-nearest-neighbor triplet pairing interactions. However, we could not ignore the triplet pairing interactions between electrons with parallel spins at high fields where the singlet pairing is suppressed by the Pauli pairbreaking effect.

It might be considered that the contributions to the triplet pairing interactions from $\bar{\chi}_{2}>0$ may be completely canceled by the larger negative contributions from $\bar{\chi}_{1}<0$. In order to illustrate that an attractive channel remains as a total, let us consider an example in which there are only two kinds of $\chi_{i j}$ with opposite signs, and write them $\bar{\chi}_{1}$ and $\bar{\chi}_{2}$. For example, in a one-dimensional tight binding model, if we truncate $\chi_{i j}$ at the nextnearest-neighbor sites, we obtain $\bar{\chi}_{1}<0$ and $\bar{\chi}_{2}>0$ and $\left|\bar{\chi}_{1}\right|>\left|\bar{\chi}_{2}\right|$.

Then, the gap equations are written in a matrix form

$$
\left(\begin{array}{c}
\Delta_{1} \\
\Delta_{2}
\end{array}\right)=-V\left(\begin{array}{ll}
\bar{\chi}_{1} W_{11} & \bar{\chi}_{1} W_{12} \\
\bar{\chi}_{2} W_{21} & \bar{\chi}_{2} W_{22}
\end{array}\right)\left(\begin{array}{c}
\Delta_{1} \\
\Delta_{2}
\end{array}\right)
$$

where $V=3 g / 8>0$ for the singlet pairing and $V=$ $-g / 8<0$ for the triplet pairing. The matrix elements $W_{n m}$ are defined by

$$
W_{n m}=\frac{1}{N} \sum_{\mathbf{k}} \gamma_{n}(\mathbf{k}) \frac{\tanh \frac{\epsilon_{\mathbf{k}}}{2 T}}{2 \epsilon_{\mathbf{k}}} \gamma_{m}(\mathbf{k}),
$$

where $\gamma_{n}(\mathbf{k})$ are the momentum dependent factors to expand the gap function. For example, when we consider one dimensional systems with interactions up to the nextnearest-neighbor sites, $\gamma_{n}(\mathbf{k})$ are defined by

$$
\begin{aligned}
& \gamma_{1}\left(k_{x}\right)=\sqrt{2} \cos k_{x} \\
& \gamma_{2}\left(k_{x}\right)=\sqrt{2} \cos 2 k_{x}
\end{aligned}
$$

for the singlet pairing, and

$$
\begin{aligned}
& \gamma_{1}\left(k_{x}\right)=\sqrt{2} \sin k_{x} \\
& \gamma_{2}\left(k_{x}\right)=\sqrt{2} \sin 2 k_{x}
\end{aligned}
$$

for the triplet pairing. Extensions to two dimensional systems are straightforward. For example, $\gamma_{n}$ are defined by

$$
\begin{aligned}
& \gamma_{1}(\mathbf{k})=\cos k_{x}-\cos k_{y} \\
& \gamma_{2}(\mathbf{k})=\cos 2 k_{x}-\cos 2 k_{y}
\end{aligned}
$$

for a $d$-wave-like pairing, while they are defined by

$$
\begin{aligned}
& \gamma_{1}(\mathbf{k})=\sqrt{2} \sin k_{x} \\
& \gamma_{2}(\mathbf{k})=2 \sin k_{x} \cos k_{y}
\end{aligned}
$$

for a $p$-wave-like pairing. The matrix elements $W_{12}=$ $W_{21}$ are not equal to zero for non-half-filling.

Now, we show that the two eigen values of the matrix on the right hand side of eq. (4) have opposite signs in general. They are equal to

$$
\begin{aligned}
\lambda_{ \pm} & =\frac{1}{2}\left[\left(\bar{\chi}_{1} W_{11}+\bar{\chi}_{2} W_{22}\right)\right. \\
& \pm \sqrt{\left(\bar{\chi}_{1} W_{11}+\bar{\chi}_{2} W_{22}\right)^{2}-4 \bar{\chi}_{1} \bar{\chi}_{2}\left(W_{11} W_{22}-W_{12}^{2}\right)} .
\end{aligned}
$$

Since $\bar{\chi}_{1} \bar{\chi}_{2}<0$ and $W_{11} W_{22}-W_{12}^{2}>0$, the second term in the square root is positive and thus the two eigen values $\lambda_{ \pm}$have opposite signs. $W_{11} W_{22}-W_{12}^{2}>0$ can be proved as follows. First, we consider an average

$$
\langle\cdots\rangle \equiv \frac{1}{N} \sum_{\mathbf{k}} \frac{\tanh \frac{\epsilon_{\mathbf{k}}}{2 T}}{2 \epsilon_{\mathbf{k}}}(\cdots) .
$$

Then, since an inequality $\left\langle\left(\gamma_{1} x-\gamma_{2}\right)^{2}\right\rangle>0$ is satisfied for arbitrary real number $x$, we obtain

$$
\left\langle\gamma_{1} \gamma_{2}\right\rangle^{2}-\left\langle\gamma_{1}{ }^{2}\right\rangle\left\langle\gamma_{2}{ }^{2}\right\rangle<0
$$

Thus, $W_{11} W_{22}-W_{12}^{2}>0$ is proved.

The fact that the two eigen values have opposite signs means that both the singlet and triplet interactions have attractive channels. For antiferromagnetic fluctuations, the attractive triplet interaction is weaker than the attractive singlet interaction as we discussed above. Thus, usually, the singlet pairing should occur at zero field. However, at high fields where the singlet pairing is suppressed, the triplet pairing takes place. With an assumption that magnetic field of the order of $\mu_{e} H \sim \Delta_{0}$ does not change the feature of the spin fluctuations drastically, we could predict a phase diagram as shown in Fig. 1] on a $T$ - $H$ plane. 


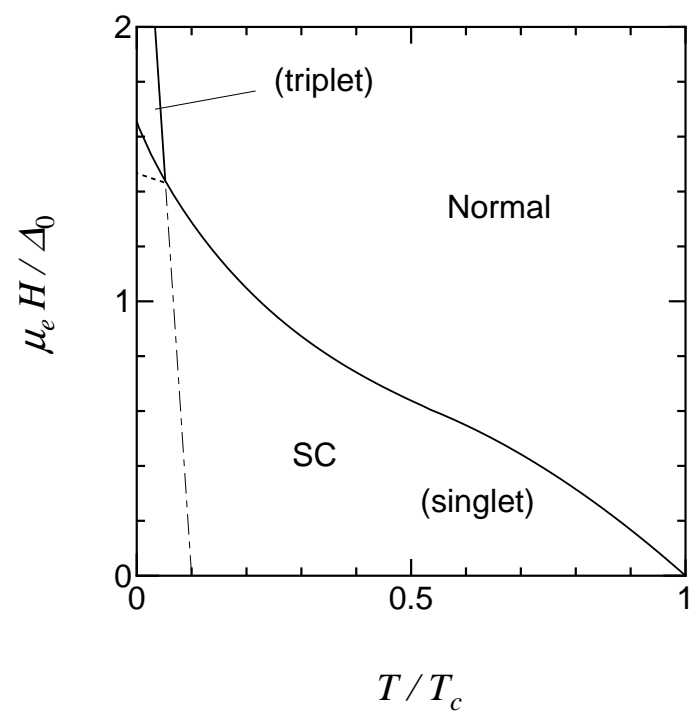

FIG. 1. Schematic phase diagram on a temperature and magnetic field plane. The vertical solid line at high fields shows the transition temperature of a triplet superconductivity of parallel spin pairing. The solid curve shows the critical field of singlet superconductivity, which is lowered into the dotted line below the critical field of the triplet superconductivity at low temperatures. The dotted-broken line is the transition temperature of the triplet superconductivity when the singlet pairing is suppressed.

Since the magnetic field is parallel to the highly conductive layers, the orbital pair-breaking effect is not efficient. Thus, the transition temperature of the triplet superconductivity of parallel spin pairing is reduced only slightly by the magnetic field. Hence, the triplet superconductivity appears at high fields.

The upturn of the critical field $\left(\partial^{2} H_{c} / \partial T^{2}>0\right)$ of singlet superconductivity at low temperatures in Fig.1 is due to an appearance of Fulde-Ferrell-Larkin-Ovchinnikov state (FFLO state or LOFF state) 17, 18]. In low dimensional systems, the FFLO state is enhanced by Fermisurface effects at low temperatures [19,20]. A reentrant transition to a triplet superconductivity might be observed at higher fields [21], but the transition temperature could recover only up to the zero field value at most.

Our schematic phase diagram is consistent with the experimental results in (TMTSF $)_{2} \mathrm{ClO}_{4}$ 22 and (TMTSF $)_{2} \mathrm{PF}_{6}$ [23]. The observed critical field also shows an upturn at low temperatures $\left(\partial^{2} H_{c} / \partial T^{2}>0\right)$, and tends to increase divergently in a low temperature limit $\left(\partial H_{c} / \partial T \rightarrow-\infty\right)$, although the observated transitions have widths and are rather ambiguous.

In the FFLO state, the singlet and the triplet order parameters are mixed in general, since the finite centerof-mass momentum q of the pairs breaks a symmetry in the real space, and at the same time the magnetic field breaks the rotational symmetry in the spin space. Hence, the FFLO state includes a component of a triplet superconductivity of antiparallel spin pairing. Matsuo et al. examined the mixing of the order parameters in an FFLO state, and found that the critical field is remarkably enhanced 24]. In particular, the tri-critical temperature of the normal, BCS, FFLO phase is enhanced.

The lower critical fields of the FFLO state and the tricritical point are not drawn in Fig.11, since it is difficult to assert their locations. It is also difficult to predict the orders of the transitions at the lower critical fields. In (TMTSF $)_{2} \mathrm{ClO}_{4}$, since first order transitions below the upper critical fields have not been observed, the FFLO state might be suppressed in this compound by impurity scatterings or some other reasons. In such a situation, the upturn of the critical field does not occur in our phase diagram. Such a suppression of the FFLO state might be a reason of the widths of the transitions at the upper critical fields in (TMTSF) ${ }_{2} \mathrm{ClO}_{4}$.

In our phase diagram, we should be careful about the choice of the ratio of the transition temperatures of the singlet pairing and the triplet pairing at zero field. The transition temperatures are extremely sensitive to the strengths of the pairing interactions, Coulomb repulsive interactions, impurity scatterings for anisotropic pairings, and so on. For example, if the triplet pairing interactions are too weak although they exist, triplet superconductivity at high fields is not observed in practice.

On the other hand, the result of the recent Knight shift measurements in (TMTSF $)_{2} \mathrm{PF}_{6} \sqrt{13}$ might be explained by taking a larger ratio of the transition temperatures. In this compound, triplet superconductivity is supported by the Knight shift measurements in a strong magnetic field $B=2.38[\mathrm{~T}]$, and the superconducting transition temperature was still about $40 \%$ of the zero field transition temperature $T_{\mathrm{c}}{ }^{(0)}=1.18[\mathrm{~K}]$ in spite of the high magnetic field. If the superconductivity at zero field is due to a singlet pairing mediated by antiferromagnetic fluctuations, the strong magnetic field $B=2.38[\mathrm{~T}]$ may favor a triplet pairing, since the pairing interactions include attractive triplet channels.

We have illustrated the existence of an attractive triplet channel in the interactions mediated by antiferromagnetic fluctuations in restricted cases. However, it is plausible that this feature is general. For example, the interactions between distant sites were calculated in a perturbation theory based on the quasi-one-dimensional Hubbard model, and it was found that triplet pairing interactions can be attractive [7].

In a 1/4-filled Hubbard model with electron dispersion

$$
\epsilon_{\mathbf{k}}=-2 t \cos k_{x} a-2 t^{\prime} \cos k_{y} b-\mu,
$$

a perturbation theory within an RPA gives pairing interactions $V\left(\mathbf{R}_{i j}=m \mathbf{a}+n \mathbf{b}\right) \equiv V_{m n}$ shown in Table [I and II, where $\mathbf{a}$ and $\mathbf{b}$ are lattice vectors. In these tables, we find oscillations of the interactions in the real space. For triplet pairing, for example, $V_{10}<0$ and $V_{40}<0$ are attractive interactions. The former favors the intrachain nearest-neighbor component of the gap function $\Delta_{10} \sin \left(k_{x} a\right)$, which does not have a node on the open 
Fermi-surfaces. On the other hand, the latter favors the gap function of the form $\Delta_{40} \sin \left(4 k_{x} a\right)$, which has line nodes on the Fermi-surface near $\mathbf{k}=( \pm \pi / 4 a, \pm \pi / 2 b)$ for $1 / 4$-filling. In practice, a superposition up to more distant components gives the gap function localized around the Fermi-surfaces with a width related to the spin fluctuations.

TABLE I. Singlet pairing interactions $V_{n m} / t$ in a quasi-one-dimensional Hubbard model with $t^{\prime}=0.150 t$ and $\underline{U} \approx 0.148 t$.

\begin{tabular}{r|rrr}
\hline \hline & $n=0$ & $n=1$ & $n=2$ \\
\hline$m=0$ & 2.769 & -0.067 & 0.020 \\
1 & 0.181 & 0.006 & -0.002 \\
2 & -0.465 & 0.087 & -0.031 \\
3 & -0.084 & -0.004 & 0.004 \\
4 & 0.252 & -0.102 & 0.036 \\
5 & 0.056 & -0.002 & -0.005 \\
$\cdots$ & & $\cdots \cdots$ & \\
\hline \hline
\end{tabular}

TABLE II. Triplet pairing interactions $V_{n m} / t$ in a quasi-one-dimensional Hubbard model with $t^{\prime}=0.150 t$ and $\underline{U} \approx 0.148 t$.

\begin{tabular}{r|rrr}
\hline \hline & $n=0$ & $n=1$ & $n=2$ \\
\hline$m=0$ & 0.768 & 0.024 & -0.013 \\
1 & -0.078 & -0.002 & 0.001 \\
2 & 0.187 & -0.031 & 0.010 \\
3 & 0.032 & 0.002 & -0.001 \\
4 & -0.100 & 0.036 & -0.012 \\
5 & -0.020 & 0.001 & 0.002 \\
$\cdots$ & & $\cdots \cdots$ & \\
\hline \hline
\end{tabular}

In conclusion, we point out that the pairing interactions mediated by antiferromagnetic fluctuations necessarily accompany attractive channels of triplet pairing interactions. Therefore, in quasi-low-dimensional type II superconductors in magnetic fields parallel to the highly conductive layers, if the origin of the pairing interactions is the exchange of antiferromagnetic fluctuations, a triplet pairing occurs at high fields where the singlet pairing is suppressed by the Pauli pair-breaking effect. We propose a schematic phase diagram which may explain the experimental observation in (TMTSF $)_{2} \mathrm{ClO}_{4}$.

We have discussed quasi-low-dimensional systems in a strong parallel magnetic field. However, if some extra mechanism suppresses the singlet pairing more than the triplet pairing, antiferromagnetic fluctuations can give rise to a triplet pairing superconductivity even at zero field, or even in three dimensional systems. For example, intersite Coulomb repulsions may suppress the pairing between electrons on nearest- and next-nearest-neighbor sites. Also, the suppression of anisotropic superconductivity due to impurity scatterings would be different depending on the pairing anisotropy. The quasi-onedimensional organic superconductors (TMTSF) ${ }_{2} \mathrm{X}$ have open Fermi-surfaces. Thus, the triplet pairing of the form $\Delta(\mathbf{k}) \sim \sin \left(k_{x} a\right)$, which was discussed by Belin et al. [12], does not have a node on the Fermi-surface. Such a nodeless gap function might have some advantage in comparison to the other anisotropic gap functions with line nodes. These possibilities will be examined in a separate paper.

This work was supported by a grant for CREST from JST.

[1] V. J. Emery: Synth. Met. 13 (1986) 21.

[2] M. T. Beal-Monod, C. Bourbonnais, and V. J. Emery: Phys. Rev. B 34 (1986) 7716.

[3] D. J. Scalapino, E. Loh, Jr., and J. E. Hirsch: Phys. Rev. B 34 (1986) 8190.

[4] K. Miyake, S. Schmitt-Rink, C. M. Varma: Phys. Rev. B 34 (1986) 6554.

[5] K. Miyake, T. Matuura, K. Sano, and Y. Nagaoka: J. Phys. Soc. Jpn. 57 (1988) 722.

[6] H. Shimahara, S. Takada: J. Phys. Soc. Jpn. 57 (1988) 1044.

[7] H. Shimahara, J. Phys. Soc. Jpn. 58 (1989) 1735; H. Shimahara, Proceeding of the Physics and Chemistry of Organic Superconductors, edited by G. Saito and S. Kagoshima (Springer-Verlag, Berlin, Heidelberg, New York, 1990), p.73.

[8] K. Yonemitsu: J. Phys. Soc. Jpn. 59 (1990) 2183.

[9] K. Ueda, T. Moriya and Y. Takahashi: J. Phys. Soc. Jpn. 59 (1990) 2905; J. Phys. Chem. Solids (UK), 53, (1992) 1515.

[10] K. Ishida et al.: Nature 396 (1998) 658. 
[11] G. M. Luke et al.: Nature 394 (1998) 558.

[12] For example, S. Belin and K. Behnia, Phys. Rev. Lett. 79 (1997) 2125.

[13] Lee et al.: cond-mat/0001332

[14] M. Takigawa, H. Yasuoka, and G. Saito: J. Phys. Soc. Jpn. 56 (1987) 873.

[15] Y. Hasegawa and H. Fukuyama: J. Phys. Soc. Jpn. 56 (1987) 877.

[16] H. Shimahara: cond-mat/9912134.

[17] P. Fulde and R. A. Ferrell: Phys. Rev. 135 (1964) A550.

[18] A. I. Larkin and Yu. N. Ovchinnikov: Zh. Eksp. Teor. Fiz. 47 (1964) 1136; translation: Sov. Phys. JETP, 20 (1965) 762 .

[19] H. Shimahara: Phys. Rev. B 50 (1994) 12760; J. Phys. Soc. Jpn. 66 (1997) 541; J. Phys. Soc. Jpn. 68 (1999) 3069.

[20] M. Miyazaki, K. Kishigi, and Y. Hasegawa: condmat/9908488.

[21] A. G. Lebed', Pis'ma Zh. Eksp. Teor. Fiz. 44 (1986) 89; translation: JETP Lett. 44 (1986) 114; Phys. Rev. B 59 (1999) R721.

[22] I. J. Lee, A. P. Hope, M. J. Leone and M. J. Naughton: Synth. Met. 70 (1995) 747.

[23] I. J. Lee, M. J. Naughton, G. M. Danner and P. M. Chaikin, Phys. Rev. Lett. 78 (1997) 3555.

[24] S. Matsuo, H. Shimahara, and K. Nagai: J. Phys. Soc. Jpn. 63 (1994) 2499. 